\title{
Effect of Different Weed Control Methods on Growth and Yield Attributing Characters of Kalmegh (Andrographis paniculata Nees)
}

\author{
Bolta Ram Meena ${ }^{1 *}$, Sunita T. Pandey ${ }^{2}$, Shiv Singh Meena ${ }^{3}$, \\ Subhashis Praharaj ${ }^{4}$ and D.C. Kala ${ }^{5}$ \\ ${ }^{1}$ Department of Agronomy, ${ }^{2}$ Department of Soil Science, GBPUA\&T Pantnagar, \\ Uttrakhand, India \\ *Corresponding author
}

\section{A B S T R A C T}

Keywords

Medicinal,

Aromatic,

Kalmegh,

Pendimethalin,

Quizalofop ethyl.

Article Info

Accepted:

19 April 2017

Available Online:

10 May 2017
Medicinal and aromatic plants play significant role in the life of people and contribute significantly to rural economy and health security of the country. Kalmegh is an important medicinal plant that has been effectively used in traditional Asian medicines for centuries. Weed infestation is the major constraint for cultivation of kalmegh in the country. If we controlled weeds efficiently then increase yield and quality of kalmegh. Keeping in view, an experiment was conducted at the Medicinal Plants Research and Development Centre, GB Pant University of Agriculture and Technology, U.S. Nagar (Uttarakhand), India during the kharif season. The experimental treatments consisted of application of herbicide (Pendimethalin and Quizalofop ethyl), mulch and Hand weeding either single or in combination along with control. The crop was transplanted on silty clay loam soil, having $\mathrm{pH} 7.3$ to study the effect of different weed control methods on growth and herbage yield of kalmegh. Application of different weed control methods in kalmegh which significant increase in crop biomass yield.

\section{Introduction}

Medicinal and aromatic plants play a significant role in the life of people and are present in innumerable forms. It also played a significant role in many ancient Indian Systems of Medicine. Kalmegh (Andrographis paniculata Nees) is a medicinal plant that has been effectively used in traditional Asian medicines for centuries. Kalmegh belongs to family Acanthaceae. This plant is known as "Mahatita" in North India which literally means "king of bitter". The genus Andrograp his consists of 40 species and about 19 species are reported to be available in India, out of which Andrographis paniculata and Andrographis alata have medicinal properties. Kalmegh has several medicinal properties such as abortifacient, analgesic, anti-inflammatory, anti-bacterial, anti-periodic, choleratic, depurative, digestive, expectorant, hepatoprotective, hypoglycaemic, laxative and sedative properties. It is a domestic medicine for flatulence and diarrhoea of children in India. The leaves of kalmegh contain the highest amount of andrographolide. Kalmegh is a short duration crop and grown for medicinal 
purpose in kharif season and thus weed infestation is very high in this crop. Weeds deteriorate the quality and quantity of kalmegh, so weed management is very essential for maintaining the herbage yield and quality of plant. Hand weeding is best method of weed controlled if availability of labour Mirjha et al., (2013).

\section{Materials and Methods}

\section{Experimental site}

The experiment was conducted at the Medicinal Plants Research and Development Centre, GB Pant University of Agriculture and Technology, U.S. Nagar (Uttarakhand) during the kharif season. There search centre lies in the taraibelt, $30 \mathrm{~km}$ southern end of foothills of Shivalik range of Himalaya at 290 latitude $79.50 \mathrm{E}$ longitude and at an altitude of $243.83 \mathrm{~m}$ above mean sea level. Tarai belt is characterized by a sub-tropical and sub humid climate in which summer is hot and dry and winter is severe cold.

Winter season falls between OctoberFebruary, then summer season continues till the end of June. During summer, maximum temperature exceeds $400 \mathrm{C}$ while in winter, the minimum temperature occasionally touches 20C. Monsoon sets in the second or third week of June and continues up to the end of September. Some rain is also received during winter months.

The average rainfall of Pantnagar is $1420 \mathrm{~mm}$ and most of rains are received from SouthWest monsoon from June to September. The soil of the experimental area was silty clay loam in texture being low in available nitrogen (215.76 kg ha-1), high in available phosphorus (29.38kg ha-1), medium in available potassium (231kg ha-1) and high organic carbon $(0.88 \%)$ contents with near neutral in reaction $(\mathrm{pH} \mathrm{7.3)}$.

\section{Experimental design and details of treatments}

The crop was transplanted on July 15, 2013 as per the randomized complete block design with three replications. The crop was transplanted in row $40 \mathrm{~cm}$ apart and $30 \mathrm{~cm}$ withinrows. Well rooted plants of A. paniculata cv. 'CIM-Megha' of uniform size and 45 days stage were transplanted in the experimental field followed by a light irrigation. The experiment, comprising of ten treatments (Table 1) viz., T1(Pendimethalin PE@ 1 kga.i. ha-1followed by mechanical weeding by hand hoe at 30-35 DAT), T2 (Quizalofop ethyl PoE at 3-5 leaf stage of weeds @ 50 g a.i. ha-1followedby mechanical weeding by hand hoe at 30-35 DAT), T3 (Pendimethalin PE @ 1 kga.i. ha-1 + Quizalofop ethyl PoE at 3-5 leaf stage of weeds @ 50 ga.i. ha-1), T4(Pendimethalin PE @ 1 kga.i. ha-1+ Quizalofop ethyl PoE at 3-5 leaf stage of weeds @50 g a.i. ha-1followed by mechanical weeding by hand hoe at 30-35 DAT), T5(Pendimethalin PE @ 1 kg a.i. ha1+ straw mulch @ 3 t ha-1), T6 (Pendimethalin PE @ 1kga.i. ha-1 + straw mulch@ 5 t ha-1), T7 (Two hand weeding at 15-20 and 30-35 DAT),T8 (Three hand weeding at 15-20, 30-35 and 45-50 DAT), T9 (Weedy check) and T10(Weed free). The data recorded for each parameter were subjected to analysis for variance for Randomised Block Design with the help of OPSTAT programme developed by the CCSHAU, Hissar. Data were analysed using the software automatically and theanalysed data were presented in the tables. For weed population and weed dry matter, the data were transformed using square root transformation $(\mathrm{x}+1)$ and analysed as above.

\section{Observations}

The crop was harvested at 90 days after transplanting in the field. Observations on 
fresh biomass yield were recorded by cutting the crop at ground level. Observations were recorded on weeds; weed density (no./m2), weed dry matter $(\mathrm{g} / \mathrm{m} 2)$ and weed control efficiency. Observations were recorded on vegetative parameter of crop growth (plant height, number of branches plant-1, number of leaves plant-1, fresh weight plant-1, dry weight plant-1, leaf area index, crop growth rate and yield parameters; fresh herbage yield (kg ha-1) and dry biomass yield (kg ha-1).

\section{Results and Discussion}

\section{Effect on weeds}

Effect of different treatments on weed density, weed dry matter and weed control efficiency was found significant at all stages of crop growth (Table 1). Grassy, broad leaved and sedge all types of weeds were found in the experimental field consisting of 22.16, 38.02 and 39.79 per cent of total weed density at harvest under weedy check treatment Upadhyay et al., (2011). Effective control of weeds (in terms of weed density, dry matter accumulation of weeds and weed control efficiency) was achieved in weed free treatment followed by T8 (Three hand weeding), T4 (Pendimethalin PE @ 1 kg a.i. ha.-1 +Quizalofop ethyl PoE at 3-5 leaf stage of weeds @ $50 \mathrm{~g}$ a.i./ha followed by mechanical weeding by hand hoe at 30-35 DAT) and T7 (Two hand weeding) treatments. It was mainly due to all weeds were effectively controlled through hand weeding during the crop growth periods resulting into lower weed density, dry matter accumulation of weeds and increase weed control efficiency Bhullar et al., (2015), Shil and Adhikary (2015). Higher weed density, dry matter accumulation of weeds and weed control efficiency was observed in weedy check treatment. Broad leaved and grassy weeds were effectively controlled by the chemical weed control methods, but sedge weeds were not effectively controlled by the chemical weed control methods, sedge weeds were effectively controlled by manually and mechanically hand weeding Dapke et al., (2014).

\section{Effect on crop}

Effect of different treatments on crop dry matter, crop growth rate and leaf area index was found significant at all stages of crop growth (Table 2). Dry matter accumulation of crop plants increased with advanced of crop age and reached maximum at 90 DAT (harvest) Wagner and Nadasy (2006). At all stages of crop growth, maximum dry matter accumulation of crop plants was recorded in weed free treatment followed by T8 (Three hand weeding), T4 (Pendimethalin PE @ 1 kg a.i. ha-1 + Quizalofop ethyl PoE @ $50 \mathrm{~g}$ a.i. ha-1 followed by mechanical weeding) and T7 (Two hand weeding) treatment. Maximum leaf area index and crop growth rate were also recorded in weed free treatment followed by T8 (Three hand weeding) at all stages of crop growth. The lowest leaf area index and crop growth rate were obtained in weedy check treatment. It was mainly due to all weeds were effectively controlled in weed free treatment by repeated hand weeding which significantly increased leaf area index and crop growth rate Gupta et al., (2014), Tamang et al., (2014).

It is concluded on the basis of experimental results, it could be concluded that the treatment T4 (Pendimethalin PE @ 1 kg a.i. ha-1 + Quizalofop ethyl PoE @ 50 g a.i. ha-1 followed by mechanical weeding) may be taken as an alternative to the hand weeding for efficient weed control and achieving high biomass yield of kalmegh during kharif season. 
Table.1 Treatment details of the experiment

\begin{tabular}{|c|c|c|}
\hline S.no. & Treatment & Treatment details \\
\hline 1. & $\mathrm{~T}_{1}$ & $\begin{array}{l}\text { Pendimethalin PE @ } 1 \mathrm{~kg} \text { a.i. ha }{ }^{-1} \text { followed by mechanical weeding by hand hoe } \\
\text { at 30-35 DAT }\end{array}$ \\
\hline 2. & $\mathrm{~T}_{2}$ & $\begin{array}{l}\text { Quizalofop ethyl PoE at 3-5 leaf stage of weeds @ } 50 \mathrm{~g}^{\mathrm{a} . i .} \mathrm{ha}^{-1} \text { followed by } \\
\text { mechanical weeding by hand hoe at } 30-35 \text { DAT }\end{array}$ \\
\hline 3. & $\mathrm{~T}_{3}$ & $\begin{array}{l}\text { Pendimethalin PE @ } 1 \text { kga.i. ha }{ }^{-1}+\text { Quizalofop ethyl PoE at 3-5 leaf stage of } \\
\text { weeds @ 50 g a.i. ha }{ }^{-1}\end{array}$ \\
\hline 4. & $\mathrm{~T}_{4}$ & $\begin{array}{l}\text { Pendimethalin PE @ 1 kga.i. ha }{ }^{-1}+\text { Quizalofop ethyl PoE at } 3-5 \text { leaf stage of } \\
\text { weeds @ } 50 \text { ga.i. ha }{ }^{-1} \text { followed by mechanical weeding by hand hoe at } 30-35 \\
\text { DAT }\end{array}$ \\
\hline 5. & $\mathrm{~T}_{5}$ & Pendimethalin PE @ 1 kga.i. ha ${ }^{-1}+$ straw mulch @ 3 tha $^{-1}$ \\
\hline 6. & $\mathrm{~T}_{6}$ & Pendimethalin PE @ $1 \mathrm{~kg}$ a.i. ha ${ }^{-1}+$ straw mulch @ $5 \mathrm{tha}^{-1}$ \\
\hline 7. & $\mathrm{~T}_{7}$ & Two hand weedings at $15-20$ and $30-35$ DAT \\
\hline 8. & $\mathrm{~T}_{8}$ & Three hand weeding at $15-20,30-35$ and $45-50$ DAT \\
\hline 9. & $\mathrm{~T}_{9}$ & Weedy check \\
\hline 10. & $\mathrm{~T}_{10}$ & Weed free \\
\hline
\end{tabular}

$\mathrm{PE}=$ pre-emergence, $@=$ at the rate, a.i. = active ingredient, ha $=$ hectare, DAT $=$ days after transplanting, PoE $=$ postemergence, T1-Pendimethalin PE @ 1 kg a.i. ha-1 + mechanical weeding at 30-35 DAT, T2-Quizalofop ethyl PoE @ $50 \mathrm{~g}$ a.i. ha-1 + mechanical weeding at 30-35 DAT, T3-Pendimethalin PE @ 1 kga.i. ha-1 + Quizalofop ethyl PoE @ 50 g a.i. ha-1, T4Pendimethalin PE @ 1kga.i. ha-1 + Quizalofop ethyl PoE@ 50 ga.i. ha-1 + mechanical weeding at 30-35 DAT, T5-

Pendimethalin PE @ 1 kga.i. ha-1 + straw mulch @ 3 tha-1, T6-Pendimethalin PE @ 1 kg a.i. ha-1 + straw mulch @ 5 t ha-1, T7-Two hand weeding at 15-20 and 30-35 DAT, T8-Three hand weeding at 15-20, 30-35 and 45-50 DAT, T9-Weedy check, T10-Weed free

Table.2 Effect of different weed management treatment on weed density, dry matter accumulation and WCE

\begin{tabular}{|c|c|c|c|c|c|c|c|c|c|}
\hline \multirow[t]{2}{*}{$\begin{array}{l}\text { Treat } \\
\text { ment }\end{array}$} & \multicolumn{3}{|c|}{ Weed density $\left(\mathbf{n o} . / \mathbf{m}^{2}\right)$} & \multicolumn{3}{|c|}{ Dry matter accumulation $\left(\mathrm{g} / \mathrm{m}^{2}\right)$} & \multicolumn{3}{|c|}{$\begin{array}{c}\text { Weed control efficiency } \\
(\%)\end{array}$} \\
\hline & 30 DAT & 60 DAT & Harvest & 30 DAT & 60 DAT & Harvest & $\begin{array}{l}30 \\
\text { DAT }\end{array}$ & $\begin{array}{l}60 \\
\text { DAT }\end{array}$ & $\begin{array}{l}\text { Harv } \\
\text { est }\end{array}$ \\
\hline $\mathrm{T}_{1}$ & $8.4(70)$ & $7.7(58)$ & $7.2(52)$ & $5.8(32.98)$ & $5.6(31.1)$ & $9.2(85.84)$ & 43.10 & 88.27 & 55.4 \\
\hline $\mathrm{T}_{2}$ & $8.7(76)$ & $8.9(80)$ & $8.4(70)$ & $6.0(35.71)$ & $6.1(36.9)$ & $9.8(96.69)$ & 39.03 & 86.06 & 48.4 \\
\hline $\mathrm{T}_{3}$ & $7.3(52)$ & $13.3(177)$ & $12.5(157)$ & $4.7(21.64)$ & $14(204)$ & $11(129.04)$ & 63.15 & 23.23 & 31.2 \\
\hline $\mathrm{T}_{4}$ & $6.6(42)$ & $6.7(44)$ & $5.7(32)$ & $4.3(18.34)$ & $4.6(20.6)$ & $5.7(32.77)$ & 68.73 & 92.22 & 82.5 \\
\hline $\mathrm{T}_{5}$ & 11.1(124) & $13.8(190)$ & $12.9(168)$ & $6.7(44.08)$ & 14(204) & 11(142.31) & 24.8 & 22.99 & 24.2 \\
\hline $\mathrm{T}_{6}$ & 10.1(101) & $12.8(1$ & 12(145) & $5.5(29.63)$ & $11(1$ & $9.5(89.59)$ & 49.46 & 51.64 & 52.2 \\
\hline $\mathrm{T}_{7}$ & $5.5(30)$ & & & $3.4(1$ & & $8.48)$ & 80.81 & 96.13 & 84.8 \\
\hline $\mathrm{T}_{8}$ & $4.90(24)$ & & & $3.2(9.71)$ & & & 83.3 & 99.78 & 99. \\
\hline $\mathrm{T}_{9}$ & $13.4(178)$ & & $15.2(232)$ & $7.7(58.75)$ & & & 0.000 & 0.000 & 0.00 \\
\hline $\mathrm{T}_{10}$ & $1.0(0.0)$ & $1(0.0)$ & $1(0.0)$ & $1.0(0.0)$ & $1.0(0.0)$ & $1.0(0.00)$ & 100.0 & 100.0 & 100. \\
\hline $\begin{array}{l}\mathrm{CD}(\mathrm{P}= \\
0.05)\end{array}$ & 0.983 & 0.762 & 0.948 & 0.363 & 0.406 & 0.703 & 6.954 & 1.128 & 1.61 \\
\hline
\end{tabular}

WCE $=$ weed control index, $\%=$ per cent, $\mathrm{g}=$ gram, $\mathrm{m}^{2}=$ meter square, DAT $=$ days after transplanting, $\mathrm{CD}=$ critical difference, $\mathrm{T}_{1}$-Pendimethalin PE @ $1 \mathrm{~kg}$ a.i. ha ${ }^{-1}+$ mechanical weeding at 30-35 DAT, T2-Quizalofop ethyl PoE @ $50 \mathrm{~g} \mathrm{a}$ ai. ha $^{-1}+$ mechanical weeding at 30-35 DAT, T 3 -Pendimethalin PE @ 1 kga.i. ha ${ }^{-1}+$ Quizalofop ethyl PoE @ $50 \mathrm{~g} \mathrm{a.i.} \mathrm{ha}^{-1}, \mathrm{~T}_{4^{-}}$

Pendimethalin PE @ 1 kga.i. ha ${ }^{-1}+$ Quizalofop ethyl PoE@ 50 ga.i. ha ${ }^{-1}+$ mechanical weeding at 30-35 DAT, $\mathrm{T}_{5}$-Pendimethalin PE@1 kga.i. ha ${ }^{-1}+$ straw mulch @ $3 \mathrm{tha}^{-1}, \mathrm{~T}_{6}$-Pendimethalin PE@ 1 kg a.i. ha ${ }^{-1}+$ straw mulch @ $5 \mathrm{t} \mathrm{ha}^{-1}$, $\mathrm{T}_{7}-\mathrm{T}_{\text {wo hand }}$ weeding at 15-20 and 30-35 DAT, $\mathrm{T}_{8}$-Three hand weeding at 15-20, 30-35 and 45-50 DAT, $\mathrm{T}_{9}$-Weedy check, $\mathrm{T}_{10}$-Weed free. 
Table.3 Effect of different weed management treatment on crop dry matter, LAI and crop growth rate

\begin{tabular}{|l|c|c|c|c|c|c|c|c|}
\hline Treatment & \multicolumn{3}{|c|}{ Crop dry matter $\left(\mathbf{g} / \mathbf{m}^{2}\right)$} & \multicolumn{3}{c|}{ LAI } & \multicolumn{2}{c|}{$\begin{array}{c}\text { Crop growth rate } \\
\left.\text { (g/m } / \mathbf{m}^{2} / \mathbf{d a y}\right)\end{array}$} \\
\cline { 2 - 9 } & 30 DAT & 60 DAT & Harvest & $\begin{array}{c}30 \\
\text { DAT }\end{array}$ & $\begin{array}{c}60 \\
\text { DAT }\end{array}$ & Harvest & $30-60$ DAT & $60-90$ \\
& & & & & & DAT \\
\hline $\mathrm{T}_{1}$ & 4.99 & 27.663 & 34.69 & 0.431 & 0.778 & 1.789 & 6.290 & 1.947 \\
\hline $\mathrm{T}_{2}$ & 3.98 & 16.000 & 23.85 & 0.341 & 0.656 & 1.667 & 3.330 & 2.170 \\
\hline $\mathrm{T}_{3}$ & 3.54 & 13.923 & 22.44 & 0.327 & 0.592 & 1.176 & 2.880 & 2.360 \\
\hline $\mathrm{T}_{4}$ & 5.25 & 31.067 & 40.18 & 0.695 & 1.568 & 2.937 & 7.163 & 2.523 \\
\hline $\mathrm{T}_{5}$ & 2.92 & 18.000 & 24.68 & 0.331 & 0.627 & 1.354 & 4.180 & 1.850 \\
\hline $\mathrm{T}_{6}$ & 3.38 & 19.000 & 29.56 & 0.409 & 0.811 & 1.671 & 4.330 & 2.927 \\
\hline $\mathrm{T}_{7}$ & 5.49 & 24.000 & 28.34 & 0.582 & 1.234 & 2.005 & 5.130 & 1.200 \\
\hline $\mathrm{T}_{8}$ & 5.30 & 34.500 & 52.81 & 0.757 & 2.092 & 3.060 & 8.103 & 5.080 \\
\hline $\mathrm{T}_{9}$ & 2.60 & 7.000 & 10.54 & 0.266 & 0.520 & 1.122 & 1.220 & 0.980 \\
\hline $\mathrm{T}_{10}$ & 5.72 & 36.440 & 59.18 & 0.823 & 3.391 & 3.944 & 8.517 & 6.310 \\
\hline $\mathrm{CD} \mathrm{P}=0.05)$ & 0.75 & 2.116 & 7.21 & 0.032 & 0.264 & 0.322 & 0.465 & 1.032 \\
\hline
\end{tabular}

$\mathrm{LAI}=$ leaf area index, $\mathrm{g}=$ gram, $\mathrm{m}^{2}=$ meter square, $\mathrm{DAT}=$ days after transplanting, $\mathrm{CD}=$ critical difference

\section{References}

Bhullar, M.S., Kaur, S., Kaur, T. and Jhala, J.A. 2015. Integrated weed management in potato using straw mulch and atrazine. Hort. Technol., 25(3): 335-339.

Dapke, S., Lambat, A., Gadewar, R., Charjan, S. and Dongre, V. 2014. Effect of different herbicides on weed control and yield in soybean (Glycine max L.). Int. J. Life Sci, Special issue A2, pp. 62-64.

Gupta, V., Singh, M., Kumar, A., Sharma, B.C. and Kher, D. 2014. Effect of different weed management practices in urdbean (Vignamungo) under sub-tropical rainfed condition. Legume Res., 37(4): 424-429.

Mirjha, P.R., Prasad, S.K., Singh, M.K., Paikra, R.H., Patel, S. and Majumdar, M. 2013. Effect of weed control measures on weeds, nodulation growth and yield of mung bean (Vignaradiata). Indian $J$. Agron., 58(4): 615-617.

Shil, S. and Adhikary, P. 2015. Weed management in transplanted chilli. Indian J. Weed Sci., 46(3): 261-263.

Tamang, D., Nath, R. and Sengupta, K. 2014. Effect of herbicide application on weed management in green gram (Vignaradiata L.). Adv. Crop Sci. Tech., 3(2): 23-29.

Upadhyay, R.K., Baksh, H., Patra, D.D., Tewari, S.K., Sharma, S.K. and Katiyar, R.S. 2011. Integrated weed management of medicinal plants in India. Int. J. Medicinal Aromatic Plant, 1(2): 51-56.

Wagner, G. and Nadasy, E. 2006. Effect of preemergence herbicide on growth parameters of green pea. Commu. Agric. Appl. Biol. Sci., 71(3): 809.

Bolta Ram Meena, Sunita T. Pandey, Shiv Singh Meena, Subhashis Praharaj and Kala, D.C. 2017. Effect of Different Weed Control Methods on Growth and Yield Attributing Characters of Kalmegh (Andrographis paniculata Nees). Int.J.Curr.Microbiol.App.Sci. 6(5): 2152-2156. doi: https://doi.org/10.20546/ijcmas.2017.605.241 